\title{
Disentangling the Imaginary-Time Formalism at Finite Temperature
}

\author{
S.M.H. Wong \\ School of Physics and Astronomy, University of Minnesota, Minneapolis, Minnesota 55455
}

\begin{abstract}
We rewrite the imaginary-time formalism of finite temperature field theory in a form that all graphs used in calculating physical processes do not have any loops. Any production of a particle from a heat bath which is itself not thermalized or the decay and absorption of a similar particle in the bath is expressed entirely in terms of the sum of particle interaction processes. These are themselves very general in meaning. They can be straight forward interactions or the more subtle and less well-known purely interference processes that do not have a counterpart in the vacuum.
\end{abstract}

PACS: 11.10.Wx, 12.38.Bx, 12.38.Mh

NUC-MINN-00/08-T

\section{INTRODUCTION}

For equilibrium field theory at finite temperature, there are two main methods of performing calculations. They are the imaginary-time formalism where one starts out in Euclidean space and analytically continues back into Minkowski space at the end of the calculation, and the real-time formalism where the calculation is done in Minkowski space with real time throughout. Because the latter has explicit real time dependence, it is therefore more suitable for time-dependent problems. However it also has the feature of the doubling of the field degrees of freedom so that each field acquired a partner and the propagators became $2 \times 2$ matrices, therefore in the sense that the components used in calculations are scalar quantities at $T=0$ versus matrix quantities in the real-time formalism the simple analogy and straightforward similarity to field theoretical calculation in the vacuum are absent. Intuitively, the difference between the calculation at zero and finite temperature should only be that the latter acquires thermal weights in the phase space integrations. This clearly is not the case in the real-time formalism because one has in addition to deal with matrix quantities. In this regard, the imaginary-time formalism resembles much more the zero temperature field theory. Having said that it must be stated that we are well aware of the Braaten-Pisarski resummation where perturbation theory at finite temperature must be rearranged [1] 5] so that it is not just a matter of thermal weights between zero and finite temperature. There are various applications of this resummation scheme, see for example the above references and also [6 [10]. These have also been recast into the form of kinetic equations for soft particles within a heat bath 11 14. We will choose not

\footnotetext{
*Here we are considering everything in the zero temperature field theory as scalar quantities, in other words for the purpose of discussion here, we make no distinctions between scalar, spinor, and vector fields related quantities. These are all scalar in the sense they have no doubling of the degrees of freedom in the vacuum.
}

to complicate matters in this paper and leave resummation out, not even including it partially as in 15 18, or in other words we will consider only particles typically at the same scale as the temperature or higher when resummation is formally not necessary. We will address the imaginary-time formalism itself and deal with a number of issues on its usage. Because of its intrinsic similarity with the vacuum theory, it should be possible to bring it into the form where the difference is essentially in the thermal weights. It will be shown, however, that there is a limit to how far this resemblance will go. And we will go even further by cutting all internal loops.

The imaginary-time formalism is a very nice formalism in that by following the established calculational rules of replacing the thermal discrete imaginary energy sum by contour integrals and analytic continuation, the calculations can be done very similarly to the familiar vacuum field theory [19]. The needed thermal distributions will be there at the end and repeated self-energy insertion along a single line will be sorted out even if that line is subsequently cut or put on-shell provided the rules are followed correctly. Thus the formalism is very compact which can hide many physical processes. For this reason, although the formalism is quite superior mathematically, it can be very unclear when it comes to finding out exactly what physical processes are involved in a particular calculation. The mathematical advantage becomes the disadvantage when it comes to the physics. For each given calculation involving medium modification of the properties of a particle or the production of one, it should be possible to write the result in a form that is a sum of all the individual contributing processes. These should apparently be the phase space integral of each relevant process weighed by a product of thermal distributions but this is not entirely true as has already been mentioned and will be clear later.

Another related aspect in thermal field theory that has not been categorically pointed out and discussed is the much richer possibility of interference when a process happens inside a heat bath. Although interference graphs are well-known to be necessary both at zero and non-zero temperature, they have largely kept their $T=0$ form when being discussed within the finite $T$ context [20,21]. Thus much of the richness was not obvious and remained 
hidden within the "simple" zero temperature Feynman graphs. The authors are only aware of infrequent mentions of this here and there, see ref. 22, 23 which are two of the few papers which, as far as the aspects of the possibility of interference is concerned, had gone to some depths. This may be the case because the existence of spectator particles easily masks any forward scattering or similar processes and so ensuring that it is impossible to tell whether the latter happened or not. When we say forward scattering here, we mean it in a generalized sense because this can happen to both a fermion or a boson. We will discuss this below, pursue further in the direction of 22, 23 but within the imaginary-time formalism and use thermal QED coupled to a massive vector particle which is itself not part of the heat bath as an example in Sect. VI.

\section{THERMAL PROCESS = VACUUM PROCESS \& THERMAL WEIGHTS, ALMOST BUT NOT QUITE}

In thermal calculations, it very often concerns the rate of production of some particles from the heat bath which are themselves not thermalized or how the medium modifies the properties of a particle such as its decay or absorption inside the hot medium. When this is done within the imaginary-time formalism, one can calculate this order by order (again we are ignoring resummation here) and the formalism will yield formulae involving thermal distributions and other expressions. This is fine if one is interested only in the answer. If on the other hand, one would like to know what physical processes contribute to the production of a particle or how another get absorbed or stimulated to decay inside a medium, one can express the formalism in another more physically explicit way but equivalent to the original formalism. To this end, we now makes the claim that the differential production or emission rate of a particle with 4-momentum $k$ per unit volume per unit time from a heat bath, but which is itself not in the heat bath, is essentially given by the discontinuity of the self-energy as

$$
2 k^{0} \frac{d R}{d^{3} k}=-\frac{i \operatorname{Disc} \Pi(k)}{\exp \left(k^{0} / T\right)-1}=I(k)+J(k)
$$

for a scalar boson (the generalization to vector bosons is straight forward) and

$$
2 k^{0} \frac{d R}{d^{3} k}=-\frac{i \operatorname{Disc} \Sigma(k)}{\exp \left(k^{0} / T\right)+1}=I(k)+J(k)
$$

for a fermion. Each of these can be explicitly expressed in terms of a sum of all the physical processes contributing to the production of this particle represented by the function $I(k)$ and $J(k)$ on the right-hand-side (r.h.s.). The $I(k)$ part, because of its resemblance to what one gets by cutting rules at zero temperature 24, 25, is known (see below). It is essentially the sum of the relevant phase space integrals over the allowed kinematical range of the probability for each production process weighed by the particle distributions. The existence of the $J(k)$ part is, however, not well-known at least not to the same extent that it will be expressed later on in this paper. It is very easy therefore to assume erroneously that there is only the contribution from $I(k)$. This is the case for example in the papers 20,26] We will clarify this in the following sections.

For those processes that do not involve spectator interference graph (see Sect. V for clarification) and therefore whose probabilities can be written as squared modulus of the corresponding matrix elements, they are represented by

$$
\begin{aligned}
I(k)= & \sum_{\mathcal{P}} \int d \Phi_{\mathcal{P}}(2 \pi)^{4} \delta^{4}\left(-k+\sum_{i \in \mathcal{P}} s_{i} p_{i}\right) \\
& \times\left|\mathcal{M}_{\mathcal{P}}\right|^{2} F_{\mathcal{P}}
\end{aligned}
$$

Here in the energy-momentum conserving delta function for each process $\mathcal{P}$, there are the signs $s_{i}$ which depend on whether each 4 -momentum $p_{i}$ is incoming $s_{i}=+$ or outgoing $s_{i}=-$. $F_{\mathcal{P}}$ is a product of particle distributions for each of the participants that has an entry in the energy-momentum conserving delta function

$$
F_{\mathcal{P}}=\prod_{i \in \mathcal{P}} s_{i}\left(\theta\left(s_{i}\right)+\mathfrak{s}_{i} \theta\left(-s_{i}\right)\right) f^{\left(\mathfrak{s}_{i}\right)}\left(p_{i}^{0}\right),
$$

where we used $f^{(+)}$and $f^{(-)}$to denote Bose-Einstein and Fermi-Dirac distribution respectively and $\mathfrak{s}_{i}$ is a sign for this purpose. The measure $d \Phi_{\mathcal{P}}$ represents that of the phase space integrations of the process $\mathcal{P}$

$$
d \Phi_{\mathcal{P}}=\prod_{i \in \mathcal{P}} \frac{d^{4} p_{i}}{(2 \pi)^{4}} \delta^{\left(s_{i}\right)}\left(p_{i}^{2}-m_{i}^{2}\right) .
$$

We have used this $I(k)$ above and will use it again below for generic representation of this kind of sum of the contributing processes of the squared modulus type.

The other generic function $J(k)$ is for the sum of interference processes that involve spectator particles in either one probability amplitude of the convoluting pair and the emission-absorption or vice versa of the same particles in the other amplitude of the pair. The function can be represented by

$$
\begin{aligned}
J(k)= & \sum_{\mathcal{P}^{\prime}} \int d \Phi_{\mathcal{P}^{\prime}}^{\prime} d \boldsymbol{\varphi}_{\mathcal{P}^{\prime}}(2 \pi)^{4} \delta^{4}\left(-k+\sum_{i \in \mathcal{P}^{\prime}} s_{i} p_{i}\right) \\
& \times\left(\overline{\mathcal{M}}_{\mathcal{P}^{\prime}} \mathcal{M}_{\mathcal{P}^{\prime}}^{*}+\overline{\mathcal{M}}_{\mathcal{P}^{\prime}}^{*} \mathcal{M}_{\mathcal{P}^{\prime}}\right) F_{\mathcal{P}^{\prime}} \mathcal{F}_{\mathcal{P}^{\prime}} .
\end{aligned}
$$

Because in these interference graphs, there are emissionabsorption of particles of the same momentum in a single amplitude and some of these possibilities have their origin in thermal self-energy insertions on the external lines, which can also be viewed as generalized forward 
scattering on the external lines, the phase space integration measure $d \Phi_{\mathcal{P}^{\prime}}^{\prime}$ for such a typical process $\mathcal{P}^{\prime}$ in general becomes

$$
\begin{aligned}
d \Phi_{\mathcal{P}^{\prime}}^{\prime}= & \prod_{i \in \mathcal{P}^{\prime}, i \notin \mathcal{S}} \frac{d^{4} p_{i}}{(2 \pi)^{4}} \delta^{\left(s_{i}\right)}\left(p_{i}^{2}-m_{i}^{2}\right) \\
& \times \prod_{j \in \mathcal{S}} \frac{d^{4} p_{j}}{(2 \pi)^{4}}(-1)^{n_{j}} \delta^{\left(n_{j}\right)\left(s_{j}\right)}\left(p_{j}^{2}-m_{j}^{2}\right)
\end{aligned}
$$

for a subgroup $\mathcal{S}$ of all the external lines of the process $\mathcal{P}^{\prime}$ in which each member $j$ in the subgroup has $n_{j}$ number of thermal self-energy insertions. We find it clearer here to say thermal self-energy insertion since this should be familiar to the readers, but we will eventually switch to the new meaning of the occurrence of any generalized forward scattering on the external line $j$. This will be further explained below in Sect. N. The above phase space integrations only take care of those momenta that enter into the overall energy-momentum conserving delta function. There are other momentum integrations that usually being labeled as loop-momenta. These are represented by the other measure $d \boldsymbol{\varphi}_{\mathcal{P}^{\prime}}$ in Eq. (6) above. Here it suffices to state that we do not consider loops as such because each loop can be opened up and interpreted as emission-absorption of particles with the same 4-momentum. This interpretation will allow us to treat these loop-momentum integrals as phase space integrals so the measure $d \boldsymbol{\varphi}_{\mathcal{P}^{\prime}}$ can be expressed in general in a form very similar to Eq. (7). These other phase space integrations will therefore acquire thermal distributions as well. The product of these is $\mathcal{F}_{\mathcal{P}^{\prime}}$ in Eq. (6). This is different from $F_{\mathcal{P}^{\prime}}$ which retains the form in Eq. (₫) for the external lines.

For the decay or absorption of a particle with momentum $k$ and mass $M$ not thermalized in the medium, we can similarly write

$$
2 M \Gamma=+\frac{i \operatorname{Disc} \Pi(k)}{\exp \left(-k^{0} / T\right)-1}=I(-k)+J(-k)
$$

for a boson and

$$
2 M \Gamma=-\frac{i \operatorname{Disc} \Sigma(k)}{\exp \left(-k^{0} / T\right)+1}=I(-k)+J(-k)
$$

for a fermion with the same mass. We are treating the absorption as stimulated decay in the presence of the medium, hence the width here $\Gamma$ is the width in the medium. The thermal factor in the denominators of Eqs. (11), (2), (8) and (9) are correct because in the limit $T \longrightarrow 0$, there is no longer a medium to produce any particle so $d R / d^{3} k \longrightarrow 0$. The width, however, will remain finite and is given now by the discontinuity of the self-energy in the vacuum.

\section{SIMPLE EXAMPLES}

The relation of the discontinuity of the self-energy to the sum of phase space integrals over each contributing process given in the previous section can be readily shown to hold for simple cases. We review the case of the oneloop self-energy as a simple example. For the production of a massive fermion from other massless fermions and bosons in a heat bath, the required loop graph is the one of the usual fermion self-energy. Within the imaginarytime formalism, we write it as

$$
\Sigma(k)=T \sum_{p_{4}=i p_{0}} \int \frac{d^{3} p}{(2 \pi)^{3}} \frac{\mathcal{N}(k, p)}{p^{2}(k-p)^{2}} .
$$

Here we have not stated explicitly which theory is being considered except by using the usual fermion self-energy graph, it has to be one with vector coupling between boson and fermion-antifermion. The numerator is simply denoted by a function $\mathcal{N}$ since its details are not required here. After converting the discrete energy sum into contour integration and performing the latter [19], one gets

$$
\begin{aligned}
\Sigma(k) & =-\int \frac{d^{3} p}{(2 \pi)^{3}} \\
& \times\left\{\left.\frac{\left(\frac{1}{2}+f^{(+)}(p)\right)}{2 p} \frac{\mathcal{N}(k, p)}{(k-p)^{2}}\right|_{p_{0}=p}\right. \\
& +\left.\frac{\left(\frac{1}{2}+f^{(+)}(p)\right)}{2 p} \frac{\mathcal{N}(k, p)}{(k-p)^{2}}\right|_{p_{0}=-p} \\
& +\left.\frac{\left(\frac{1}{2}-f^{(-)}(|\boldsymbol{k}-\boldsymbol{p}|)\right)}{2|\boldsymbol{k}-\boldsymbol{p}|} \frac{\mathcal{N}(k, p)}{p^{2}}\right|_{p_{0}=k_{0}+|\boldsymbol{k}-\boldsymbol{p}|} \\
& \left.+\left.\frac{\left(\frac{1}{2}-f^{(-)}(|\boldsymbol{k}-\boldsymbol{p}|)\right)}{2|\boldsymbol{k}-\boldsymbol{p}|} \frac{\mathcal{N}(k, p)}{p^{2}}\right|_{p_{0}=k_{0}-|\boldsymbol{k}-\boldsymbol{p}|}\right\} .
\end{aligned}
$$

The vacuum part has also been included in the above expression. Now performing analytic continuation and taking the discontinuity of the self-energy $\Sigma(k)$ with the delta function $\delta\left(k_{0}+p+|\boldsymbol{k}-\boldsymbol{p}|\right)$ for the production of the massive fermion in mind, only the second and the third term in Eq. (11) have the right discontinuity. For production, we have to set $k^{0} \longrightarrow-k^{0}$ to get

$$
\begin{aligned}
i \operatorname{Disc} \Sigma(k) & =-\int \frac{d^{3} p}{(2 \pi)^{3}}(2 \pi) \delta\left(-k_{0}+p+|\boldsymbol{k}-\boldsymbol{p}|\right) \\
\times & \left.\frac{\left(1+f^{(+)}(p)-f^{(-)}(|\boldsymbol{k}-\boldsymbol{p}|)\right)}{2 p 2|\boldsymbol{k}-\boldsymbol{p}|} \mathcal{N}(-k, p)\right|_{p_{0}=p} .
\end{aligned}
$$

This can be rearranged to

$$
\begin{aligned}
&-\frac{i \operatorname{Disc} \Sigma(k)}{\exp \left(k_{0} / T\right)+1} \\
&=\int \frac{d^{4} p}{\left(2 \pi^{\prime}\right)^{4}}(2 \pi) \delta^{(+)}\left(p^{2}\right) \frac{d^{4} p^{\prime}}{\left(2 \pi^{\prime}\right)^{4}}(2 \pi) \delta^{(+)}\left(p^{\prime 2}\right) \\
& \times(2 \pi)^{4} \delta^{(4)}\left(-k+p+p^{\prime}\right) \\
& \times f^{(+)}\left(p_{0}\right) f^{(-)}\left(p_{0}^{\prime}\right) \mathcal{N}(-k, p) \\
&=\left.\quad 2 k_{0} \frac{d R}{d^{3} k}\right|_{\text {one-loop }},
\end{aligned}
$$


the form we expected from Eq. (2). The $\mathcal{N}$ is essentially just the squared of the vector-fermion-antifermion coupling, which is all there is for the squared modulus of the probability amplitude at leading order.

One could easily use Eq. (11) to obtain the discontinuity for the decay of a massive fermion into a fermion and a boson in the heat bath. The energy conserving delta function to get from Eq. (11) in this case is $\delta\left(k_{0}-p-|\boldsymbol{k}-\boldsymbol{p}|\right)$. Only the first and last term from Eq. (11) contribute. It is simple to follow the same steps and arrive at the form given in Eq. (9) or

$$
\begin{aligned}
& -\frac{i \operatorname{Disc} \Sigma(k)}{\exp \left(-k_{0} / T\right)+1} \\
= & \int \frac{d^{4} p}{\left(2 \pi^{\prime}\right)^{4}}(2 \pi) \delta^{(+)}\left(p^{2}\right) \frac{d^{4} p^{\prime}}{\left(2 \pi^{\prime}\right)^{4}}(2 \pi) \delta^{(+)}\left(p^{2}\right) \\
\times & \times(2 \pi)^{4} \delta^{(4)}\left(k-p-p^{\prime}\right) \\
& \times\left(1+f^{(+)}\left(p_{0}\right)\right)\left(1-f^{(-)}\left(p_{0}^{\prime}\right)\right) \mathcal{N}(k, p) \\
= & \left.2 M \Gamma\right|_{\text {one-loop }} .
\end{aligned}
$$

Note that this could equally have been written with $\delta^{(-)}$ with $f$ as given in the previous section instead of $\delta^{(+)}$ with $1 \pm f$. Both of the above explicit examples have not the $J(k)$ function part because this will come at higher orders. More precisely these contributions come in at two-loop and higher orders.

\section{GENERAL PROOF}

In this section in order to put up a framework so that later sections can be more readily understood, we show in our own way that Eqs. (11), (2), (8) and (9) hold in general. We saw in Sec. II that both the $I(k)$ and $J(k)$ functions possess the same structure of $I(k)$ at least for the external lines included in the delta function conserving the overall 4-momentum. That is as far as the part associated with lines of the contributing graphs that carry part of the total energy-momentum, the two functions are the same. $J(k)$ has some extra phase space integrals and distributions but these have little to do with the overall momentum conservation and they can be viewed as substructures. This will be discussed later on. First the main structure of Eqs. (12), (2), (8) and (9), which is the structure of the function $I(k)$, will be shown first.

For a self-energy at arbitrary order in the coupling of the theory, it can always be rearranged into the form shown in Fig. 11 where momentum $p_{1}$ flows through line $1, p_{2}$ flows through line $2 \ldots$ etc and the last line $m$ carries the boson or fermion momentum $k$ of the selfenergy under consideration and the sum of the other lines above it so that $p_{m}=k+\sum_{i}^{m-1} p_{i}$. The two blobs shaded differently need not be the same in general and they can be any graphs from very simple to very complex connecting the lines 1 to $m$. Our aim is to put all these lines on-shell so that they become the external lines and each has an entry in the overall 4-momentum conserving delta function. In other words, we are dividing the selfenergy in two by cutting through line 1 to $m$. There are of course more than one way to group lines into the form in Fig. 1 and therefore different cuts possible on that graph. Also at any given order, the self-energy will be a sum of graphs of the form of Fig. 1. The full results must therefore be a sum both over different graphs and cuts on those graphs. This sum will eventually become our sum over different contributing processes.

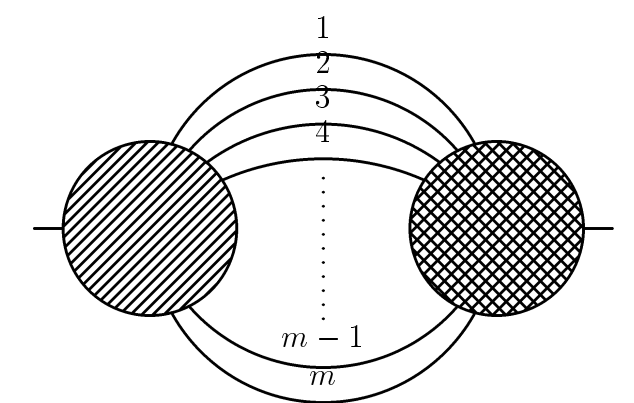

FIG. 1. A self-energy diagram at any order in the coupling can always be rearranged in this form with momentum $p_{i}$ flowing through line $i$ except the last line $m$ where the momentum $p_{m}=k+\sum_{i}^{m-1} p_{i}$. The two blobs may be complex vertices or $(m+1)$-point Green's functions and they need not be the same, hence the different shadings. There are of course more than one possibility of such an arrangement for each graph.

\section{A. The Case of All Bosonic External Lines}

Our own approach to arrive at the results in Eqs. (1), (2), (8) and (9) is by using the imaginary-time formalism and performing the contour integrals of the loop momenta $p_{1}, p_{2} \ldots p_{m-1}$, without loss of generality, in that order. Furthermore, lines 1 to $m$ will all be taken to be bosonic and massless for simplicity. The case that some lines are bosonic and some fermionic is a generalization which does not affect the proof provided equations to be used below are suitably modified. This will be touched upon briefly in Sect. IVB. Since the discontinuity of the self-energy contains a number of different processes, we will aim at getting only one process, say that with the overall energy-momentum delta function

$$
\delta^{(4)}\left(k+\sum_{i}^{m} s_{i} p_{i}\right)
$$

where the $s_{i}$ 's is a fixed set of signs associated with this particular process depending on which momentum $p_{i}$ is outgoing or incoming. We adopt the convention that $s=+$ for incoming or absorption of a particle and $s=-$ for outgoing or emission. This applies also to $k$. By concentrating on Eq. (15), any pole in the contour integrations that does not contribute to this particular chosen process need not be picked up and will be dropped from the discussion. For each line, which one of the two poles 
can be picked is determined by the signs $s_{i}$ 's so that there is only one pole per line that will contribute to Eq. (15). Starting from the $p_{1}^{0}$ contour integration, it can either pick the pole on line 1 or that on line $m$. In either case, the $p_{2}^{0}$ integration will have also two possibilities, that of line 2 or line $m$ in the former case and line 2 or line 1 in the latter. Since each contour integration will have two possibilities to pick a pole so that there will be $2^{m-1}$ terms that contribute to Eq. (15) after all $m-1$ contour integrations have been performed. Picking a pole on a line in a contour integral is equivalent to putting that line on-shell and giving it a factor of a thermal distribution. Since there are $m-1$ integrations and $m$ lines, all but one line will remain off-shell at the end. This line will be cut and put on-shell as well when the discontinuity of the self-energy is finally taken. Therefore although in general the numerators of the $2^{m-1}$ terms will be different after the integrations because different sets of external lines may have been put on-shell, they will become identical once the discontinuity has been taken. We take the numerator $\mathcal{N}\left(k, p_{1}, p_{2}, \ldots, p_{m-1}\right)$ as the part that does not include the thermal distributions and is evaluated at the mass-shells of these particles. Because it is the same for every term, it can be taken out of the following discussion as a common factor. We can concentrate entirely on manipulating the products of thermal distributions into the desired form. Those readers not interested in the details of this manipulation could accept on faith that the final form of the product of distributions is correct and skip to section B to only examine the identities and the simple examples in section $\mathrm{C}$.

After the discontinuity has been taken, there will be $2^{m-1}$ terms, each with a different product of thermal distributions. Writing the propagators in the convenient form

$$
\frac{1}{p_{0 i}^{2}-p_{i}^{2}}=\frac{1}{2 p_{i}} \sum_{s_{i}= \pm} \frac{s_{i}}{p_{0 i}-s_{i} p_{i}}
$$

for $i=1$ to $m-1$ and

$$
\frac{1}{p_{0 m}^{2}-p_{m}^{2}}=\frac{1}{2 p_{m}} \sum_{s_{m}= \pm} \frac{-s_{m}}{p_{0 m}+s_{m} p_{m}}
$$

where $p_{i}=\left|\boldsymbol{p}_{i}\right|$ for $i=1, \ldots, m-1$ and $p_{m}=\mid \boldsymbol{k}+$ $\sum_{i}^{m-1} \boldsymbol{p}_{i} \mid$. If the $p_{1}^{0}$ contour integration picks up the pole on line 1 , there will be the factor $-\left(1 / 2+f^{(+)}\left(s_{1} p_{1}\right)\right)$ which includes also the vacuum part. On the other hand if it is the pole on line $m$ that is picked, then there will instead be $\left(1 / 2+f^{(+)}\left(-s_{m} p_{m}\right)\right)$. In the first case, subsequent integration of $p_{2}^{0}$ will yield either the $-\left(1 / 2+f^{(+)}\left(s_{2} p_{2}\right)\right)$ or $\left(1 / 2+f^{(+)}\left(-s_{1} p_{1}-s_{m} p_{m}\right)\right)$ thermal factor depending on whether the pole on line 2 or line $m$ is picked. In the other case, there will be $-\left(1 / 2+f^{(+)}\left(s_{2} p_{2}\right)\right)$ or $\left(1 / 2+f^{(+)}\left(-s_{1} p_{1}-s_{m} p_{m}\right)\right)$ from line 2 and line 1 respectively. Continuing like so, there will be different products of thermal distributions. Starting from the case where each contour integration picks the pole of its own line, then only one of the lines 1 to $m-1$ picks the pole of line $m$ instead of its own line, next two lines do not pick their own lines and so on until none of the $p_{i}^{0}$ contour integration pick their own pole at $p_{i}^{0}=s_{i} p_{i}$. Using the simplifying notations,

$$
\begin{gathered}
f_{i}^{(+)}=f^{(+)}\left(s_{i} p_{i}\right) \\
f_{-j}^{(+)}=f^{(+)}\left(-s_{j} p_{j}\right) \\
f_{m+i+j+\cdots}^{(+)}=f^{(+)}\left(+s_{m} p_{m}+s_{i} p_{i}+s_{j} p_{j}+\cdots\right), \\
f_{-m-i-j-\cdots}^{(+)}=f^{(+)}\left(-s_{m} p_{m}-s_{i} p_{i}-s_{j} p_{j}-\cdots\right),
\end{gathered}
$$

and

$$
\tilde{f}_{i}^{(+)}=1 / 2+f_{i}^{(+)}
$$

etc. The sum of thermal factors from the $2^{m-1}$ terms with all common factors taken out can therefore be written as

$$
\begin{aligned}
& \mathfrak{F}=\prod_{i}^{m-1} \tilde{f}_{i}^{(+)}-\sum_{j=1}^{m-1}\left(\prod_{i \neq j}^{m-1} \tilde{f}_{i}^{(+)}\right) \tilde{f}_{-m-\sum_{i \neq j}^{(+)} i}^{(+)} \\
& +\sum_{j=1}^{m-2} \sum_{k=j+1}^{m-1}\left(\prod_{i \neq j \neq k}^{m-1} \tilde{f}_{i}^{(+)}\right) \tilde{f}_{-m-\sum_{i \neq j}^{(+)} i}^{(+)} \tilde{f}_{-m-\sum_{i=1}^{k-1} i}^{(+)} \\
& -\sum_{j=1}^{m-3} \sum_{k=j+1}^{m-2} \sum_{l=k+1}^{m-1}\left(\prod_{i \neq j \neq k \neq l}^{m-1} \tilde{f}_{i}^{(+)}\right) \tilde{f}_{-m-\sum_{i \neq j}^{j-1} i}^{(+)} \\
& +\ldots \tilde{f}_{-m-\sum_{i=1}^{k-1} i}^{(+)} \tilde{f}_{-m-\sum_{i=1}^{l-1} i}^{(+)} \\
& +(-1)^{m-1} \tilde{f}_{-m}^{(+)} \tilde{f}_{-m-1}^{(+)} \tilde{f}_{-m-1-2}^{(+)} \tilde{f}_{-m-1-2-3}^{(+)} \cdots \cdots \\
& \quad \times \tilde{f}_{-m-1-2-\cdots-(m-2)}^{(+)} .
\end{aligned}
$$

The key to finding a way out of this seemingly endless sum of terms is the observation that each term must have a partner. By that we mean for every term containing $\tilde{f}_{m-1}^{(+)}$as part of the thermal weight, there must be another one that differs from this term only in this factor by having $-\tilde{f}_{-m-1-2-\cdots-(m-2)}^{(+)}$in its place instead. This is true because no matter which sets of poles were picked in the contour integrations, the last integration of $p_{m-1}^{0}$ must be able to pick either its own pole on line $m-1$ or the pole on the other line that now carries the energy $p_{m-1}^{0}$. This could be the line $m$ if it has not yet been touched or another line with the energy $p_{m-1}^{0}$ shifted there via an already performed contour integration. Therefore every term must have either $\tilde{f}_{m-1}^{(+)}$or $-\tilde{f}_{-m-1-2-\cdots-(m-2)}^{(+)}$by virtue of the last contour integration $p_{m-1}^{0}$. They can thus all be paired. Now using the delta function Eq. (15), we get the identity 


$$
\begin{aligned}
& \tilde{f}_{m-1}^{(+)}-\tilde{f}_{-m-1-2-\cdots-(m-2)}^{(+)} \\
& =f_{m-1}^{(+)}-f_{-m-1-2-\cdots-(m-2)}^{(+)} \\
& =\left(e^{-k^{0} / T}-1\right) f_{m-1}^{(+)} f_{m+1+2+\cdots+(m-2)}^{(+)}
\end{aligned}
$$

After this is applied to every pair, the common factor on the r.h.s. of Eq. (24) can be taken out of Eq. (23). We now have $2^{m-2}$ terms to sort out.

Shifting focus now onto the thermal weight $\tilde{f}_{m-2}^{(+)}$or $-\tilde{f}_{-m-1-2-\cdots-(m-3)}^{(+)}$. For similar reason as before, each term must have either one of the two and these again form pairs because of the second last contour integration of $p_{m-2}^{0}$. The identity

$$
\begin{aligned}
& \left(f_{m-2}^{(+)}-f_{-m-1-2-\cdots-(m-3)}^{(+)}\right) f_{m+1+2+\cdots+(m-2)}^{(+)} \\
& =f_{m-2}^{(+)} f_{m+1+2+\cdots+(m-3)}^{(+)}
\end{aligned}
$$

is a more general form of Eq. (24) and can be used to remove the last factor in the now common thermal factor on the r.h.s. of Eq. (24) to get the new common factor

$$
\left(e^{-k^{0} / T}-1\right) f_{m-1}^{(+)} f_{m-2}^{(+)} f_{m+1+2+\cdots+(m-3)}^{(+)} .
$$

The number of terms has now been further reduced to $2^{m-3}$. Iterating this thermal factor reduction process, all $2^{m-1}$ terms can be grouped together eventually into one common thermal factor

$$
\begin{aligned}
\mathfrak{F}= & \left(e^{-k^{0} / T}-1\right) f_{m}^{(+)} f_{m-1}^{(+)} f_{m-2}^{(+)} f_{m-3}^{(+)} \cdots \cdots \\
& \times \cdots \cdots f_{3}^{(+)} f_{2}^{(+)} f_{1}^{(+)}
\end{aligned}
$$

The first factor will be divided out by the denominator in Eq. (11) with $k^{0} \longrightarrow-k^{0}$ for production or that in Eq. (8) for decay. The remaining thermal distributions will be $f^{(+)}(p)$ for absorption with $s=+$ in the delta function in Eq. (15) or $-\left(1+f^{(+)}(p)\right)$ for emission when $s=$ - as expected. Combining the sign factors $s_{1} s_{2} \cdots s_{m}$ originating from the propagators in Eq. (18) but have been left out of the discussion so far, this is the $F_{\mathcal{P}}$ in Eqs. (3) and (4)

$$
F_{\mathcal{P}}=\frac{s_{1} s_{2} \cdots s_{m}}{e^{-k^{0} / T}-1} \mathfrak{F}
$$

Now we turn to the phase space integrals in Eq. (3). From the $m-1$ loop integrations, there are already the 3-momentum measures $d^{3} p_{i} /(2 \pi)^{3}$ for $i=1$ to $m-1$. This together with the $1 /\left(2 p_{i}\right)$ from the propagators give $d \Phi$ in Eq. (5) from $i=1$ up to the $m-1$ entry. The last entry $m$ can be gotten by introducing the identity

$$
\int \frac{d^{3} p_{m}}{(2 \pi)^{3}}(2 \pi)^{3} \delta^{(3)}\left(k+\sum_{i=1}^{m} s_{i} p_{i}\right)=1 .
$$

Multiplying this by the remaining $1 / 2 p_{m}$ and the energy delta function $(2 \pi) \delta\left(k^{0}+\sum_{i=1}^{m} p_{i}^{0}\right)$ from the discontinuity gives

$$
\int \frac{d^{4} p_{m}}{(2 \pi)^{4}}(2 \pi) \delta^{\left(s_{m}\right)}\left(p_{m}^{2}\right) \delta^{(4)}\left(k+\sum_{i=1}^{m} s_{i} p_{i}\right)
$$

By grouping everything else not discussed above which is mainly the numerator $\mathcal{N}\left(k, p_{1}, p_{2}, \cdots, p_{m}\right)$ together, this will be $\left|\mathcal{M}_{\mathcal{P}}\right|^{2}$ if in this particular process the two blobs in Fig. 1 contain no internal loop momentum that runs completely inside the blob. If either one or both contain internal loops then this will be the product of $\left(\overline{\mathcal{M}}_{\mathcal{P}} \mathcal{M}_{\mathcal{P}}^{*}+\right.$ $\left.\overline{\mathcal{M}}_{\mathcal{P}}^{*} \mathcal{M}_{\mathcal{P}}\right)$ and $\mathcal{F}_{\mathcal{P}}$ in the function $J(k)$. The latter $\mathcal{F}_{\mathcal{P}}$ factor will carry essentially the hidden loop momentum integrations and there will be associated thermal weights. We will however adopt an approach in which there will not be any loop. But this and the remaining parts of the function $J(k)$ are the subjects of Sect. V.

\section{B. The Case of a Mixture of Bosonic and Fermionic External Lines}

For the more general case where there is a mixture of fermionic and bosonic lines amongst the lines 1 to $m$ in Fig. 1, the proof is somewhat more complicated because bosonic distribution can turn into fermionic distribution and vice versa when they are evaluated at a pole depending on whether this has an imaginary part with a total of an integer $n$ or a half-integer $n+1 / 2$ of $(2 \pi i)$ imaginary energy. For a bosonic self-energy, there must be an even number of intermediate fermion lines and for a fermion self-energy, there must be an odd number of such lines. In the latter case in order not to have a contradiction in our momentum arrangement in Fig. 1, the last line $m$ must be fermionic whereas in the bosonic self-energy there is no such constraint. We could nevertheless arrange all fermion lines to be those at the bottom and leaving all boson lines at the top in either case. That is if there are $n$ boson lines $n \leq m$, we arrange the lines so that line 1 to $n$ will be bosonic and line $n+1$ to $m$ will be fermionic. Then for a fermion self-energy the last contour integration will give the pair combination

$$
\begin{aligned}
& -\tilde{f}_{m-1}^{(-)}+\tilde{f}_{-m-1-2-\cdots-(m-2)}^{(+)} \\
& =f_{m-1}^{(-)}+f_{-m-1-2-\cdots-(m-2)}^{(+)} \\
& =-\left(e^{-k^{0} / T}+1\right) f_{m-1}^{(-)} f_{m+1+2+\cdots+(m-2)}^{(+)}
\end{aligned}
$$

if the line $m-1$ is fermionic or

$$
\begin{aligned}
& -\tilde{f}_{m-1}^{(+)}+\tilde{f}_{-m-1-2-\cdots-(m-2)}^{(-)} \\
& =-f_{m-1}^{(+)}-f_{-m-1-2-\cdots-(m-2)}^{(-)} \\
& =-\left(e^{-k^{0} / T}+1\right) f_{m-1}^{(+)} f_{m+1+2+\cdots+(m-2)}^{(-)}
\end{aligned}
$$

if this line is bosonic. This is always true because the number of fermion lines connecting the two blobs in Fig. 1 is odd. Here we have used the notation $\tilde{f}_{i}^{(-)}=1 / 2-f_{i}^{(-)}$. For a bosonic self-energy, there is the combination 


$$
\begin{aligned}
& \tilde{f}_{m-1}^{(-)}-\tilde{f}_{-m-1-2-\cdots-(m-2)}^{(-)} \\
& =-f_{m-1}^{(-)}+f_{-m-1-2-\cdots-(m-2)}^{(-)} \\
& =\left(e^{-k^{0} / T}-1\right) f_{m-1}^{(-)} f_{m+1+2+\cdots+(m-2)}^{(-)} .
\end{aligned}
$$

The thermal factors can be sorted into the desire form in Eq. (4) using the identities

$$
\begin{gathered}
-\left(f_{i}^{(-)}-f_{-j}^{(-)}\right) f_{i+j}^{(+)}=f_{i}^{(-)} f_{j}^{(-)} \\
\left(f_{i}^{(+)}+f_{-j}^{(-)}\right) f_{i+j}^{(-)}=f_{i}^{(+)} f_{j}^{(-)} \\
-\left(f_{i}^{(-)}+f_{-j}^{(+)}\right) f_{i+j}^{(-)}=f_{i}^{(-)} f_{j}^{(+)}
\end{gathered}
$$

iteratively as similarly done before in the previous section. The phase space integration measure and the rest are the same as discussed there. The proof of the main form of the function $I(k)$ or $J(k)$ is therefore complete. In Sect. V, the integrand and substructures in $I(k)$ and $J(k)$ or equivalently the internal structures of the blobs in Fig. 11 will be the subject.

\section{Examples}

In this subsection, we give two examples of the thermal factor reduction from $2^{m-1}$ terms down to one discussed in the previous section. First we consider a bosonic selfenergy with $m=3$ and one-fermion loop. In accordance to our discussions above, we push all fermion lines to the bottom so line 1 will be boson and line 2 and 3 will be fermion. Without stating explicitly what process to get from the self-energy, we choose the very general energy conserving delta function $\delta\left(k_{0}+s_{1} p_{1}+s_{2} p_{2}+s_{3} p_{3}\right)$. After the discontinuity has been taken, the sum of thermal distributions is

$$
\begin{aligned}
F^{\prime}=s_{1} s_{2} s_{3}( & -\tilde{f}_{1}^{(+)} \tilde{f}_{2}^{(-)}+\tilde{f}_{-3}^{(-)} \tilde{f}_{2}^{(-)} \\
& \left.+\tilde{f}_{1}^{(+)} \tilde{f}_{-1-3}^{(-)}-\tilde{f}_{-3}^{(-)} \tilde{f}_{-1-3}^{(-)}\right) .
\end{aligned}
$$

This can be reduced by pairing as follows.

$$
\begin{aligned}
F^{\prime} & =s_{1} s_{2} s_{3}\left(\tilde{f}_{1}^{(+)}-\tilde{f}_{-3}^{(-)}\right)\left(-\tilde{f}_{2}^{(-)}+\tilde{f}_{-1-3}^{(-)}\right) \\
& =s_{1} s_{2} s_{3}\left(f_{1}^{(+)}+f_{-3}^{(-)}\right)\left(f_{2}^{(-)}-f_{-1-3}^{(-)}\right) \\
& =-s_{1} s_{2} s_{3} f_{1}^{(+)} f_{2}^{(-)} f_{3}^{(-)}\left(e^{-k_{0} / T}-1\right)
\end{aligned}
$$

As another example, we consider a fermion self-energy with $m=4$. This time only line $m$ is fermionic. The sum of thermal distributions now is

$$
\begin{aligned}
F^{\prime}=s_{1} s_{2} s_{3} s_{4} & \left(-\tilde{f}_{1}^{(+)} \tilde{f}_{2}^{(+)} \tilde{f}_{3}^{(+)}+\tilde{f}_{-4}^{(-)} \tilde{f}_{2}^{(+)} \tilde{f}_{3}^{(+)}\right. \\
& +\tilde{f}_{1}^{(+)} \tilde{f}_{-1-4}^{(-)} \tilde{f}_{3}^{(+)}+\tilde{f}_{1}^{(+)} \tilde{f}_{2}^{(+)} \tilde{f}_{-1-2-4}^{(-)} \\
& -\tilde{f}_{-4}^{(-)} \tilde{f}_{-1-4}^{(-)} \tilde{f}_{3}^{(+)}-\tilde{f}_{-4}^{(-)} \tilde{f}_{2}^{(+)} \tilde{f}_{-1-2-4}^{(-)} \\
& \left.-\tilde{f}_{1}^{(+)} \tilde{f}_{-1-4}^{(-)} \tilde{f}_{-1-2-4}^{(-)}+\tilde{f}_{-4}^{(-)} \tilde{f}_{-1-4}^{(-)} \tilde{f}_{-1-2-4}^{(-)}\right) .
\end{aligned}
$$

Pairing as before gives

$$
\begin{aligned}
& F^{\prime}=s_{1} s_{2} s_{3} s_{4}\left(\tilde{f}_{1}^{(+)}-\tilde{f}_{-4}^{(-)}\right)\left(\tilde{f}_{2}^{(+)}-\tilde{f}_{-1-4}^{(-)}\right) \\
& \times\left(\tilde{f}_{3}^{(+)}-\tilde{f}_{-1-2-4}^{(-)}\right) \\
&=s_{1} s_{2} s_{3} s_{4}\left(f_{1}^{(+)}+f_{-4}^{(-)}\right)\left(f_{2}^{(+)}+f_{-1-4}^{(-)}\right) \\
& \times\left(f_{3}^{(+)}+f_{-1-2-4}^{(-)}\right) \\
&=s_{1} s_{2} s_{3} s_{4} f_{1}^{(+)} f_{2}^{(+)} f_{3}^{(+)} f_{4}^{(-)}\left(e^{-k_{0} / T}-1\right) .
\end{aligned}
$$

\section{THE INTERNAL STRUCTURES OF I(K) AND J(K)}

The previous sections showed how the discontinuity of the self-energy could be arranged into phase space integrations over all the external lines weighed by their respective thermal distributions. There we called any line that showed itself explicitly in Fig. 1 external and these all have entries in the overall energy-momentum conserving delta function in Eq. (3) and Eq. (6). In this section, we will deal with the remaining structures in these equations. These structures come essentially from the blobs in Fig. 11 which have not been discussed yet.

In the simplest case, each blob consists of a tree graph with all lines leading to the external lines 1 to $m$ and the line with 4-momentum $k$. This can be just a few lines at low orders or a very large tree at high orders. All internal lines' energies-momenta are fixed completely by the external lines and there is no additional integration and thermal distribution other than those of the external lines. If the two blobs are identical, then there is automatically the $\left|\mathcal{M}_{\mathcal{P}}\right|^{2}$. If they are not, then there is the interference $\overline{\mathcal{M}}_{\mathcal{P}} \mathcal{M}_{\mathcal{P}}^{*}+\overline{\mathcal{M}}_{\mathcal{P}}^{*} \mathcal{M}_{\mathcal{P}}$ with $\mathcal{F}_{\mathcal{P}}=1$. In the case that $\overline{\mathcal{M}}_{\mathcal{P}}$ and $\mathcal{M}_{\mathcal{P}}$ are of the same order in the coupling, there must also be the possibilities $\left|\mathcal{M}_{\mathcal{P}}\right|^{2}$ and $\left|\overline{\mathcal{M}}_{\mathcal{P}}\right|^{2}$ so that a larger squared modulus can be formed $\left|\overline{\mathcal{M}}_{\mathcal{P}}+\mathcal{M}_{\mathcal{P}}\right|^{2}$. For familiar field theories such as $\phi^{3}, \phi^{4}$, QED, QCD etc., tree graphs with the same number of external legs are of the same order so this grouping into a larger squared modulus is always possible. This case for $J(k)$ is rather trivial but has to be stated first for the sake of completeness before we move on to more complex cases. So non-identical blobs with no internal loop are the simplest examples of interference graphs in $J(k)$ that can actually be grouped into the class of function $I(k)$. It is the more complex interference graphs genuinely belonged to the $J(k)$ function part that are our main concern. Towards this we now turn.

In the previous paragraph, we discussed the case of the tree graphs. This type of contributions can generally be interpreted straight forwardly as scattering processes or other interactions. For cases of a few particles in the initial state, there will always be the vacuum counterpart. These are quite familiar physically. We need not 
say anymore on these. What we find less obvious and less well-known is the physical interpretation of the case that the blobs Fig. 1 have internal loops. Within the imaginary-time formalism, it is not too difficult, albeit tedious, to perform the calculation of these graphs and it is all too easy to lose the feel of what are the actual physical processes involved in any particular calculation. The nature of these graphs made it even harder because of the fact that first there is no vacuum counterpart and second they are the non-intuitive interference type. If one is not clear in one's thinking and clings on to the over-familiar vacuum picture, they could even appear to be impossible.

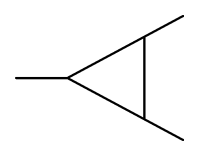

FIG. 2. An example graph for a blob with one loop.

Let us consider the simple case that one blob has one internal loop and the other none, the procedure in arriving at the form of $I(k)$ has already put all the external lines on-shell. So the calculation of this blob is equivalent to the evaluation of a one-loop $n$-point function where $n \leq(m+1)$. The rules of the imaginary-time formalism stipulated that the energy contour integration of the loop must pick out every pole in the propagators in turn of all the particles along the lines that form the loop. This step is equivalent to putting in turn every particle propagating around the loop on-shell. For each particle around the loop, there are two poles. One can be interpreted as its emission into the heat bath from one vertex and its absorption from the bath by another. The other is absorption from the heat bath now at the first vertex and its emission back into it from the second. An example graph for a blob with one internal loop and three external lines is shown in Fig. 2. This graph can be turned into the six graphs in Fig. 3 after the energy contour integration put the three internal lines in turn on mass-shell. These are indicated by the now on-shell large dotted lines in Fig. 3 .

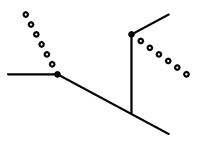

(u1)

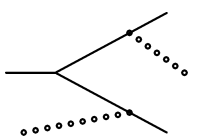

(v1)

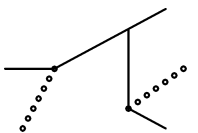

(11)

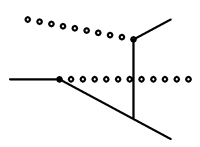

(u2)

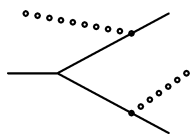

(v2)

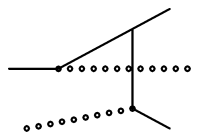

(12)
FIG. 3. Performing the energy contour integration is equivalent to putting the internal lines in turn on-shell. (u1) and (u2), (v1) and (v2), and (11) and (12) are graphs resulting from putting the upper, vertical and lower internal line respectively on-shell. Each pair of graphs corresponds to the two poles from each line.

In our special case, it is easy to turn the blob with the loop into a sum of tree graphs with emission-absorption of particles of the same momentum. If one now takes a step back and looks again at the whole picture, there is also the other blob that helps make up the whole selfenergy which consists of only a tree graph. That for our example graph in Fig. 2 will be that shown in Fig. 4 .

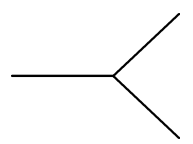

FIG. 4. The associated graph to Fig. 2.

Remembering that Fig. 2 and 4 together form an interference contribution, opening up the loop in Fig. 2 would seem to render the interference impossible because there must be the same incoming as well as outgoing particles in both graphs. However, graphs representing interactions in a heat bath are not quite the same as those in the vacuum. It is a common practice to use the same graphs in both situations but it must not be forgotten that in a heat bath graphs should be understood to be implicitly accompanied by spectator particles that made up the multiparticle system. So opening up the loop in Fig. 2 should be accompanied by a change of Fig. 1 into Fig. 5. The momentum carried by the spectator line in Fig. 5 is of course different when this is convoluted with each of the three $(\mathrm{u}),(\mathrm{v})$ and (l) pairs in Fig. 3 . 
FIG. 5. This is how Fig. 4 should be properly represented once the loop in Fig. 2 has been opened up.

So in this simple example, there will be six terms in the function $J(k)$. Each will have a phase space integration of either $s= \pm$ form

$$
d \boldsymbol{\varphi}_{\mathcal{P}^{\prime}}=\frac{d^{4} l}{(2 \pi)^{4}}(2 \pi) \delta^{(s)}\left(l^{2}-m^{2}\right)
$$

originating from the previous loop integration. Because these correspond to the emission and absorption of particles of the same momentum within a single graph, they do not play a part in the overall energy-momentum flow and have not an entry in the corresponding constraining delta function. The emission and absorption of these particles must come with thermal distribution. Because of the unusual nature of the emission-absorption or the absorption-emission in the same graph, each of these in our example with only one internal loop acquires the distribution

$$
\mathcal{F}_{\mathcal{P}^{\prime}}=1 / 2+f^{(+)}\left(\left|l^{0}\right|\right)
$$

for boson or

$$
\mathcal{F}_{\mathcal{P}^{\prime}}=1 / 2-f^{(-)}\left(\left|l^{0}\right|\right)
$$

for fermion in this simple case. For more complex cases where the blobs are not identical and each has several internal loops, after opening up each loop in the blobs and adding the corresponding spectator on the other blob the phase space integration becomes

$$
d \boldsymbol{\varphi}_{\mathcal{P}^{\prime}}=\prod_{i}^{L} \frac{d^{4} l_{i}}{(2 \pi)^{4}}(2 \pi) \delta^{\left(s_{i}\right)}\left(l_{i}^{2}-m_{i}^{2}\right)
$$

where $L$ is the combined total number of internal loops between the two blobs. If there are any self-energy insertions hidden in a blob, then this loop will have to be opened up too. Therefore each self-energy insertion will be turned into the emission and absorption of a particle of the same 4-momentum from a single line. If the line is fermionic, this is the usual forward scattering, but there is also the case that a bosonic line emits and absorbs or vice versa a fermion of the same 4 -momentum. Thus we label these generically as generalized forward scattering. In this case, the internal loop turned phase space integrations will be of the form of Eq. ( (7). The factor of thermal distribution when there are many loops becomes

$$
\mathcal{F}_{\mathcal{P}^{\prime}}=\prod_{i}^{L}\left(1 / 2+\mathfrak{s}_{i} f^{\left(\mathfrak{s}_{i}\right)}\left(\left|l^{0}\right|\right)\right) .
$$

Because there must be four or a larger even number of graphs obtainable from each loop, the sum in Eq. (6) over $\mathcal{P}^{\prime}$ is a sum over the main processes as well as over the possible subprocesses of emission-absorption of particles in each main process.
The steps in arriving at the form of the $J(k)$ part contribution to the production from or the decay of a particle in a heat bath are now shown. So expressing a production or a stimulated decay of a particle in a heat bath in terms of all the contributing processes should not be restricted only to processes expressible in terms of squared modulus amplitudes. In other words, there is the very important $J(k)$ interference contributions in addition to the better known $I(k)$ part. In the next section we will illustrate all these in an explicit example. But before we do that it must be mentioned the vacuum parts still have to be regularized and renormalized in the usual way. We have assumed that this was implicitly understood.

\section{A MORE COMPLEX AND COMPLETE EXAMPLE}

In this section we will use QED coupled to a massive vector particle as an example. The relevant lagrangian is

$$
\begin{aligned}
\mathcal{L}= & -\frac{1}{4} F^{\mu \nu} F_{\mu \nu}+\bar{\psi} \gamma^{\mu}\left(i \partial_{\mu}-e A_{\mu}-g V_{\mu}\right) \psi \\
& -\frac{1}{4} G^{\mu \nu} G_{\mu \nu}-\frac{1}{2} M^{2} V^{\mu} V_{\mu},
\end{aligned}
$$

where $F^{\mu \nu}$ and $G^{\mu \nu}$ are the field tensors for the $A^{\mu}$ and $V^{\mu}$ vector fields respectively. The coupling $g$ is taken to be much weaker than $e, e \gg g$, so only higher order corrections in $e$ will be considered. Our heat bath will consist only of leptons and photons at a temperature $T$, and the vector $V^{\mu}$ is not itself thermalized. A massive vector with a mass $M \gg T$ will be sent into the heat bath to determine the medium modification of its width up to two loops.

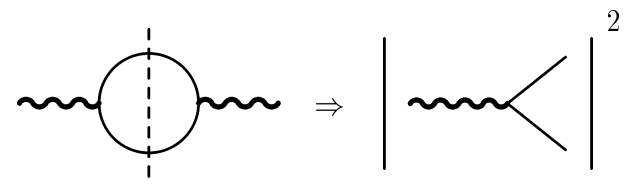

FIG. 6. The leading contribution to the decay of $V^{\mu}$.

At leading order ( $\mathrm{LO}$ ), there is only the decay into a dilepton pair contribution to Eq. (8) coming from the one-loop self-energy shown in Fig. 6. This is of course only a contribution of the type of $I(k)$. From Eq. (14) after some substitutions, this contribution is

$$
\begin{aligned}
& \left.2 M \Gamma\right|_{\mathrm{LO}} \\
= & \int \frac{d^{4} p}{(2 \pi)^{4}}(2 \pi) \delta^{(+)}\left(p^{2}\right) \frac{d^{4} p^{\prime}}{(2 \pi)^{4}}(2 \pi) \delta^{(+)}\left(p^{2}\right) \\
& \times(2 \pi)^{4} \delta^{(4)}\left(k-p-p^{\prime}\right) \\
& \times\left(1-f^{(-)}\left(p_{0}\right)\right)\left(1-f^{(-)}\left(p_{0}^{\prime}\right)\right)|\mathcal{M}|_{\mathrm{LO}}^{2} .
\end{aligned}
$$

Here the numerator is just the familiar Dirac trace of the lepton loop, one gets after summing over final spins and averaged over initial spin 


$$
|\mathcal{M}|_{\mathrm{LO}}^{2}=\frac{4 g^{2}}{3}\left\{p \cdot p^{\prime}+\frac{2(p \cdot k)\left(p^{\prime} \cdot k\right)}{M^{2}}\right\} .
$$

At the next-to-leading order, there are only three selfenergy graphs but there are many physical processes hidden within these graphs. In the following graphs, the thick wavy line is for the massive vector while the thin wavy line is for photon. The lines overlaid by the vertical dashed line are the exposed external lines of Fig. 11. First we examine those arrangements of these graphs with no internal loops within the blobs. By putting the three internal lines in the two-loop graph with an internal selfenergy in Fig. 7 on-shell, three physical processes emerge. We get a sum of the squared modulus amplitudes of two compton scattering in Fig. 8, of one decay with photon emission in Fig. 9 and of one vector-photon fusion in Fig. 10. These are clearly all contributions to the $I(k)$ function part in Eq. (8). There will of course be the other contributions where the internal self-energy is on the lower lepton line. These can easily be taken care of by a simple factor of two by symmetry.

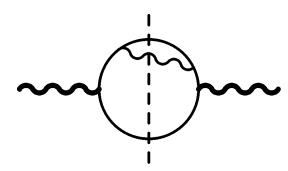

FIG. 7. Two-loop self-energy with internal self-energy insertion. All three internal lines in the middle will be put on-shell as external lines therefore the two blobs are tree graphs.

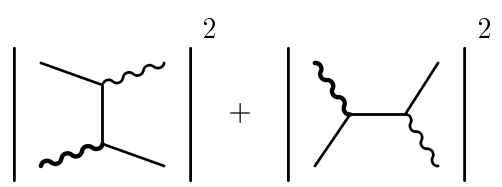

FIG. 8. Contributions to compton scattering from Fig. 7 .

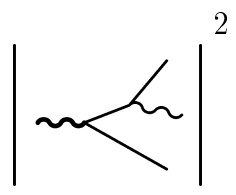

FIG. 9. Decay with photon emission from Fig. 6.

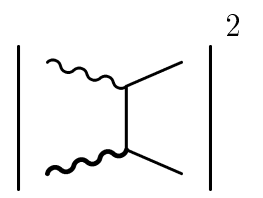

FIG. 10. Vector-photon fusion contribution from Fig. 7 .

The other two-loop graph is the one with a vertex correction drawn in Fig. 11. Putting all three internal lines on-shell, three physical, albeit interference, processes emerge. They are again compton scattering in Fig. 12, three-body decay in Fig. 13 and fusion in Fig. 14 . Because they are all interference contributions, it appears that they belong to $J(k)$. As we said in Sect. $\mathrm{V}$, blobs that have no internal loop or have only a tree structure could be regrouped with other contributions to form a larger amplitude. Examining the pairs in Fig. 8 and 12, Fig. 9 and 13 and Fig. 10 and 14 and also not forgetting that the two other lepton lines together with the photon line in Fig. 11 could also be put on-shell as well to give similar contributions, this can of course be done as is well-known. We merely broke down the contributions into parts so that they could be clearly seen, within the framework that we are presenting the paper, which contribution came from which diagram. Loosely speaking the contributions from Fig. 11 should be in $J(k)$ but it is better to tighten the definition so that this function is restricted to the genuine, not well-known in-medium interference contributions to the modification of the width.

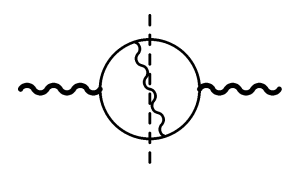

FIG. 11. Two-loop self-energy with vertex correction. Putting the three intermediate lines on-shell and there will not be any internal loop.

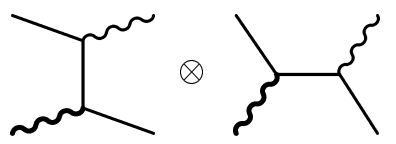

FIG. 12. Interference contribution to compton scattering from Fig. 11. This can be regrouped with Fig. 8 to form a larger squared modulus of a single amplitude. It does not genuinely belong to the $J(k)$ class.

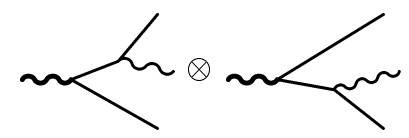

FIG. 13. Interference contribution to decay with photon emission from Fig. 11. This can also be regrouped with Fig. 9 to form a larger squared modulus of a single amplitude. This is not really a $J(k)$ class contribution.

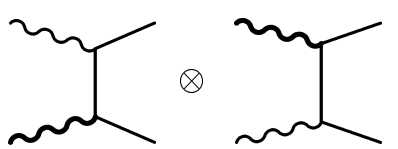

FIG. 14. Interference vector-photon fusion contribution from Fig. 11. Again together with Fig. 10 this can form a large squared modulus of a single amplitude. Therefore it is in the $I(k)$ class contribution.

After combining the contributions from Fig. 1 and 11 , we get for the three processes

$$
\begin{aligned}
&\left.2 M \Gamma\right|_{\text {compton }} \\
&=2 \int \frac{d^{4} p}{(2 \pi)^{4}}(2 \pi) \delta^{(+)}\left(p^{2}\right) \frac{d^{4} p^{\prime}}{(2 \pi)^{4}}(2 \pi) \delta^{(+)}\left(p^{2}\right)
\end{aligned}
$$




$$
\begin{aligned}
& \times \frac{d^{4} q}{(2 \pi)^{4}}(2 \pi) \delta^{(+)}\left(q^{2}\right)(2 \pi)^{4} \delta^{(4)}\left(k+p-p^{\prime}-q\right) \\
& \times f^{(-)}\left(p_{0}\right)\left(1-f^{(-)}\left(p_{0}^{\prime}\right)\right)\left(1+f^{(+)}\left(q_{0}\right)\right) \\
& \times|\mathcal{M}|_{\text {compton }}^{2}, \\
& \left.2 M \Gamma\right|_{\text {decay }} \\
= & \frac{d^{4} p}{(2 \pi)^{4}}(2 \pi) \delta^{(+)}\left(p^{2}\right) \frac{d^{4} p^{\prime}}{(2 \pi)^{4}}(2 \pi) \delta^{(+)}\left(p^{2}\right) \\
\times & \frac{d^{4} q}{(2 \pi)^{4}}(2 \pi) \delta^{(+)}\left(q^{2}\right)(2 \pi)^{4} \delta^{(4)}\left(k-p-p^{\prime}-q\right) \\
\times & \left(1+f^{(+)}\left(q_{0}\right)\right)\left(1-f^{(-)}\left(p_{0}\right)\right)\left(1-f^{(-)}\left(p_{0}^{\prime}\right)\right) \\
\times & |\mathcal{M}|_{\text {decay }}^{2}, \\
=2 \int & \left.2 M \Gamma\right|_{\text {fusion }} \\
& \quad \times \frac{d^{4} p}{(2 \pi)^{4}}(2 \pi) \delta^{(+)}\left(p^{2}\right) \frac{d^{4} p^{\prime}}{(2 \pi)^{4}}(2 \pi) \delta^{(+)}\left(p^{2}\right) \\
& \times f^{(+)}\left(q_{0}\right)\left(1-f^{(-)}\left(p_{0}\right)\right)\left(1-f^{(+)}\left(q^{2}\right)(2 \pi)^{4} \delta^{(4)}\left(k+q-p-p^{\prime}\right)\right. \\
& \times|\mathcal{M}|_{\text {fusion }}^{2} \cdot
\end{aligned}
$$

The averaged over initial spins, summed over final spins matrix elements for the three processes are

$$
\begin{gathered}
|\mathcal{M}|_{\text {compton }}^{2}=-\frac{4 e^{2} g^{2}}{3}\left\{\frac{s}{u}+\frac{u}{s}+2 t\left(\frac{1}{s}+\frac{1}{u}+\frac{t}{s u}\right)\right\} \\
|\mathcal{M}|_{\text {decay }}^{2}=\frac{8 e^{2} g^{2}}{3}\left\{\frac{p \cdot p^{\prime}+k \cdot p^{\prime}}{p \cdot q}+\frac{p \cdot p^{\prime}+k \cdot p}{p^{\prime} \cdot q}\right. \\
\left.\quad+\frac{2\left(p \cdot p^{\prime}\right)^{2}}{(p \cdot q)\left(p^{\prime} \cdot q\right)}\right\} \\
|\mathcal{M}|_{\text {fusion }}^{2}=\frac{4 e^{2} g^{2}}{3}\left\{\frac{t}{u}+\frac{u}{t}+2 s\left(\frac{1}{t}+\frac{1}{u}+\frac{s}{t u}\right)\right\} .
\end{gathered}
$$

These amplitudes were calculated in [27] for studying the change in $\mathrm{Z}$ boson properties in the quark-gluon plasma. Note that if one is only interested in the medium modification to the width, it is necessary to subtract the leading order and next-to-leading order vacuum contribution in Eqs. (47) and (50).

Now we turn to the examples of one of the main subjects of this paper. In Fig. 月 instead of putting the three intermediate lines on-shell, one could do this to two lepton lines without touching the photon as shown in Fig. 15. This results in the blob on the r.h.s. in Fig. 11 to have an internal loop made up of two lines. As we discussed already in Sect. V, they will be put on the massshell in turn by the energy contour integration. Thus it is possible to unfold the diagram into several ones with emission and absorption of particles with the same 4momentum within one graph. One must of course put in the associated spectator particles in the graph originated from the blob on the left-hand-side (LHS) of Fig. 1 in order for the new graphs to make physical sense. These are now depicted in Fig. 16 and 17. In Fig. 16, the massive vector decays into a real and virtual dilepton pair. The virtual one then either absorbed a photon from and emitted it back into the heat bath or emitted a photon into the bath before absorbing one back. In Fig. 17, the virtual lepton either annihilates with one from the heat bath to recreate another dilepton pair or it is put on-shell via a photon exchange with a lepton in the bath. The lepton line that has just been put on-shell is shown in dashed line in the figure. While all these are happening on one graph, there is merely the vector decay in the accompanying graph. Here there is a photon or a lepton spectator.

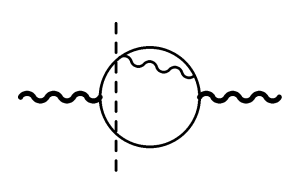

FIG. 15. In this two-loop self-energy with internal self-energy insertion, only two lines will be put on-shell. One of the blobs will have an internal loop with two lines.

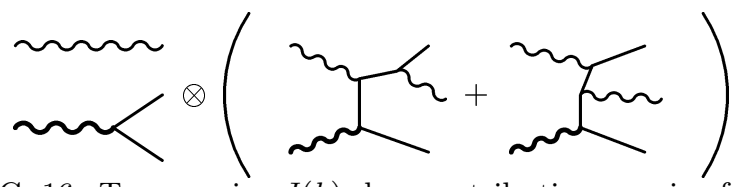

FIG. 16. Two genuine $J(k)$ class contributions coming from Fig. 15. Photon with the same 4-momentum is emitted and absorbed within a single graph. The same photon is required as a spectator in the other graph.

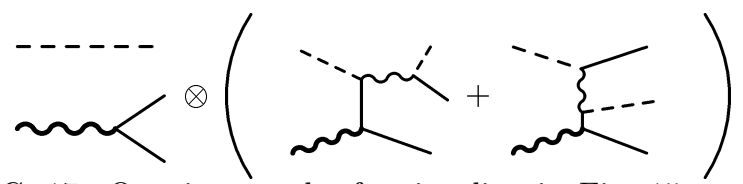

FIG. 17. Opening up the fermion line in Fig. 15 results in the absorption from and emission back of a lepton into the heat bath or vice versa. These are different examples of generalized forward scattering on the fermion line. The lepton line from the self-energy loop is now shown in dashed lines to distinguish them from the other leptons.

These interference graphs can be worked out to be

$$
\begin{aligned}
&\left.2 M \Gamma\right|_{(\text {Fig. 16) }} \\
&=2 \int \frac{d^{4} p}{(2 \pi)^{4}}(-1)(2 \pi) \delta^{(+)}\left(p^{2}\right) \frac{d^{4} p^{\prime}}{(2 \pi)^{4}}(2 \pi) \delta^{(+)}\left(p^{2}\right) \\
& \times \frac{d^{4} q}{(2 \pi)^{4}}(2 \pi) \delta^{(+)}\left(q^{2}\right)(2 \pi)^{4} \delta^{(4)}\left(k-p-p^{\prime}\right) \\
& \times\left(1-f^{(-)}\left(p_{0}\right)\right)\left(1-f^{(-)}\left(p_{0}^{\prime}\right)\right) \\
& \times\left(1 / 2+f^{(+)}\left(q_{0}\right)\right)\left(\overline{\mathcal{M}} \mathcal{M}^{*}+\overline{\mathcal{M}}^{*} \mathcal{M}\right)_{(\text {Fig. [16) }},
\end{aligned}
$$


and

$$
\begin{aligned}
&\left.2 M \Gamma\right|_{(\text {Fig. 17) }} \\
&=2 \int \frac{d^{4} p}{(2 \pi)^{4}}(-1)(2 \pi) \delta^{(+)}\left(p^{2}\right) \frac{d^{4} p^{\prime}}{(2 \pi)^{4}}(2 \pi) \delta^{(+)}\left(p^{2}\right) \\
& \quad \times \frac{d^{4} l}{(2 \pi)^{4}}(2 \pi) \delta^{(+)}\left(l^{2}\right)(2 \pi)^{4} \delta^{(4)}\left(k-p-p^{\prime}\right) \\
& \quad \times\left(1-f^{(-)}\left(p_{0}\right)\right)\left(1-f^{(-)}\left(p_{0}^{\prime}\right)\right) \\
& \quad \times\left(1 / 2-f^{(-)}\left(l_{0}\right)\right)\left(\overline{\mathcal{M}} \mathcal{M}^{*}+\overline{\mathcal{M}}^{*} \mathcal{M}\right)_{(\text {Fig. } 17.7 .} .
\end{aligned}
$$

Note that the mass-shell constraining delta function of $p$ in these equations has a derivative because of the double pole due to the double propagator $\left(1 / p^{2}\right)^{2}$. The sum over final spins and averaged over initial spins convoluted amplitudes are

$$
\begin{aligned}
&\left(\overline{\mathcal{M}} \mathcal{M}^{*}+\overline{\mathcal{M}}^{*} \mathcal{M}\right)_{(\text {Fig. } 16)} \\
&=\frac{16 e^{2} g^{2}}{3}\left\{p \cdot p^{\prime}\right.+(p \cdot q)\left(p^{\prime} \cdot q\right) \\
&\left.\times\left(\frac{1}{p^{2}+2 p \cdot q}+\frac{1}{p^{2}-2 p \cdot q}\right)\right\},
\end{aligned}
$$

and

$$
\begin{aligned}
& \left(\overline{\mathcal{M}} \mathcal{M}^{*}+\overline{\mathcal{M}}^{*} \mathcal{M}\right)_{(\text {Fig. 17) }} \\
=-\frac{8 e^{2} g^{2}}{3} & \left(2(p \cdot l)\left(p \cdot p^{\prime}\right)-p^{2}\left(p^{\prime} \cdot l\right)\right) \\
& \times\left(\frac{1}{2 p \cdot l+p^{2}}+\frac{1}{2 p \cdot l-p^{2}}\right) .
\end{aligned}
$$

Similar to what was done to Fig. 15, one can also put only two lepton lines of Fig. 11 on mass-shell as shown in Fig. 18. In this case, the blob on the r.h.s. of Fig. 1 has an internal loop consists of three lines. Converting the photon to a real one results in the photon absorptionemission interference contribution in Fig. 19. Instead of a forward scattering on the lepton in Fig. 16, now different fermions participate in the emission and absorption. Next either lepton line within the loop can be put on-shell. One gets interference contributions where the vector particle gets absorbed by a lepton before a dilepton pair is radiated off at the end and others where the vector fuses with a virtual photon radiated from a thermal lepton to form a dilepton pair. These are shown in Fig. 20. All these interfere again with the less interesting simple decay graph with a spectator.

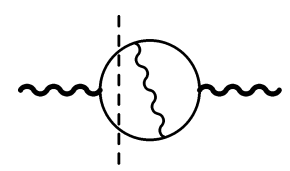

FIG. 18. Only two lines will be external lines in this two-loop self-energy and therefore one of the blobs will have an internal loop made up of three lines.

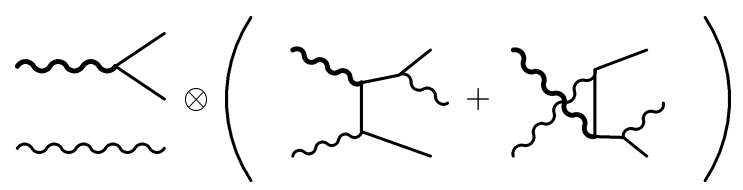

FIG. 19. From the internal loop of Fig. 18, one also gets a genuine interference contribution with photon absorption-emission within a single graph.

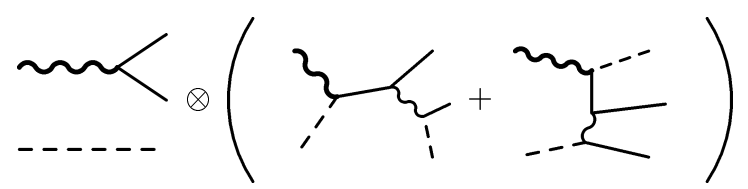

FIG. 20. A similar real interference contribution to Fig. 17 from Fig. 18. Note that the former internal but now on-shell lepton line is different from that in Fig. 18. There is another similar contribution from Fig. 18 by opening up the other lepton line in the loop.

The mathematical expressions for the contributions in Fig. 19 and 20 are similar to those in Eqs. (55) and (56) except 1) $(-1)(2 \pi) \delta^{\prime(+)}\left(p^{2}\right)$ is replaced by the usual $(2 \pi) \delta^{(+)}\left(p^{2}\right)$ and 2) the convoluted amplitudes are of course not the same. These work out to be

$$
\begin{aligned}
& \left(\overline{\mathcal{M}} \mathcal{M}^{*}+\overline{\mathcal{M}}^{*} \mathcal{M}\right)_{(\text {Fig. 19) }} \\
= & \frac{16 e^{2} g^{2}}{3}\left\{1-\frac{\left(p \cdot p^{\prime}\right)^{2}}{(p \cdot q)\left(p^{\prime} \cdot q\right)}\right\},
\end{aligned}
$$

and

$$
\begin{aligned}
& \left(\overline{\mathcal{M}} \mathcal{M}^{*}+\overline{\mathcal{M}}^{*} \mathcal{M}\right)_{(\text {Fig. } 20)} \\
= & -\frac{4 e^{2} g^{2}}{3} \frac{p^{\prime} \cdot l}{p \cdot l}\left\{\frac{p \cdot l-p \cdot p^{\prime}}{k \cdot l-p \cdot p^{\prime}}+\frac{p \cdot l+p \cdot p^{\prime}}{k \cdot l+p \cdot p^{\prime}}\right\} .
\end{aligned}
$$

The full result up to $\mathcal{O}\left(e^{2}\right)$ correction is

$$
\begin{aligned}
\Gamma= & \Gamma_{\text {LO }}+2 \Gamma_{\text {compton }}+\Gamma_{\text {decay }}+\Gamma_{\text {fusion }} \\
& +2 \Gamma_{(\text {Fig. 16) }}+2 \Gamma_{(\text {Fig. } 17)} \\
& +\Gamma_{(\text {Fig. 19 })}+2 \Gamma_{(\text {Fig. } 20)}
\end{aligned}
$$

The factor of two for the various contributions to the width is due to the two possibilities of lepton and antilepton to take part in whatever interactions that give rise to that particular contribution. Note that in the $\Gamma_{\text {decay }}, \Gamma_{\text {fusion }}$ and $\Gamma_{\text {Fig. }}$ 19, each has linear infrared divergence enhanced by the Bose-Einstein distribution but these cancel in the sum of the three [27,28].

By opening up the internal loops, each term in Eq. (61) is now a contribution from a clear physical process and not just a vague one-loop and two-loop contribution which cannot be readily associated with an interaction. In the general case of self-energy graphs at high orders so that there are multiple internal loops and overlapping lines, the imaginary-time formalism is just too compact for the physics to be transparent. In any case if one is concerned with the physical processes, a lot of terms in the self-energy will not contribute and drop out once the 
discontinuity has been taken. Therefore there will be a lot of fruitless labor, so instead of calculating hoops and loops of virtual particles, it is much simpler to disentangle all of them and calculate instead the amplitudes of onshell particle interactions. We have shown here that these could be both squared modulus processes and purely interference processes made possible by the presence of the heat bath. The latters are not so well-known and it is easy to be influenced by the accustomed vacuum picture to believe that there are only the former contributions.

Using these techniques we have, beside working out the $\mathrm{Z}$ boson width in a quark-gluon plasma [27], also calculated the high mass next-to-leading order dilepton production from such a QCD plasma. This is reported in 29]. Some of these have previously been examined in for example [18]. Since we have not included any form of resummation, we do not expect the results to agree. But there should be an agreement once the resummed version of the present work is done. We will leave this as a future work.

As a final remark although we exclusively worked within the imaginary-time formalism, the result for any of the physical quantities on the LHS in Eqs. (1), (2), (8) and (9) obtained within the real-time formalism should, of course, be the same. The connection between the real- and imaginary-time formalism for the first few $\mathrm{N}$ point functions have been worked out by various authors 20,26, 30 34. The relevant quantities in our case are the imaginary part of the two-point functions. These are related to those in the real-time formalism by

$$
\begin{aligned}
& \operatorname{Im} \Pi(k)=+\tanh \left(k_{0} / 2 T\right) \operatorname{Im} \Pi_{11}(k) \\
& \operatorname{Im} \Sigma(k)=-\operatorname{coth}\left(k_{0} / 2 T\right) \operatorname{Im} \Sigma_{11}(k) .
\end{aligned}
$$

To calculate the imaginary part of the self-energies in real-time, one can use any of the many suggested finite temperature cutting rules [20,21,23,26, 31, 35, 36]. Since we are more interested in what physical processes actually contribute to the production or decay rate of a non-thermalized particle than the mathematical rules by which one calculates the imaginary part of $\Pi(k)$ or $\Sigma(k)$, we will leave this at that and do not elaborate any further. For the details of the precise connection between any aspects of the two formalism, the readers should consult the references given here. The message that we would like to convey in this paper is that the physical processes that contribute to the LHS of Eqs. (11), (2), (8) and (9) are far richer in numbers and stranger than those found at zero temperature. The existence of a thermal bath permits many purely interference processes to contribute which have no counterpart in the vacuum. It has also been pointed out that it was possible to calculate the rates, provided that the finite temperature interference processes were included, by basing on the actual physical processes rather than on which $\mathrm{N}$-point or Nloop functions. The latter are not immediately physically transparent.

\section{ACKNOWLEDGMENTS}

The author thanks Joe Kapusta for useful discussions. This work was supported by the U.S. Department of Energy under grant no. DE-FG02-87ER40328.

[1] R.D. Pisarski, Phys. Rev. Lett. 63, 1129 (1989).

[2] E. Braaten and R.D. Pisarski, Nucl. Phys. B 337, 569 (1990).

[3] E. Braaten and R.D. Pisarski, Phys. Rev. Lett. 64, 1338 (1990).

[4] J. Frenkel and J.C. Taylor, Nucl. Phys. B 334, 199 (1990).

[5] J.C. Taylor and S.M.H. Wong, Nucl. Phys. B 346, 115 (1990).

[6] E. Braaten and M.H. Thoma, Phys. Rev. D 44, 1298 (1991).

[7] R. Baier, H. Nakkagawa, A. Niegawa, and K. Redlich, Phys. Rev. D 45, 4323 (1992).

[8] S.M.H. Wong, Z. Phys. C 53, 465 (1992).

[9] S.M.H. Wong, Z. Phys. C 58, 159 (1993).

[10] M.H. Thoma, Quark-Gluon Plasma, edited by R.C. Hwa, Vol. 2 (World Scientific 1995), p.51.

[11] J.-P. Blaizot and E. Iancu, Phys. Rev. Lett. 70, 3376 (1993).

[12] J.-P. Blaizot and E. Iancu, Nucl. Phys. B 417, 608 (1994).

[13] R. Jackiw, Q. Liu and C. Lucchesi, Phys. Rev. D 49, 6787 (1994).

[14] P.F. Kelly, Q. Liu, C. Lucchesi and C. Manuel, Phys. Rev. D 50, 4209 (1994).

[15] H.A. Weldon, Phys. Rev. D 26, 2789 (1982).

[16] J.F. Donoghue and B.R. Holstein, Phys. Rev. D 28, 340 (1983); 29 3004(E) (1984).

[17] T. Altherr, P. Aurenche and T. Becherrawy, Nucl. Phys. B 315, 436 (1989).

[18] T. Altherr and P. Aurenche, Z. Phys. C 45, 99 (1989).

[19] J.I. Kapusta, Finite Temperature Field Theory, Cambridge University Press (1989).

[20] R.L. Kobes and G.W. Semenoff, Nucl. Phys. B 272, 329 (1986).

[21] H.A. Weldon, Phys. Rev. D 28, 2007 (1983).

[22] P.V. Landshoff and J.C. Taylor, Nucl. Phys. B 430, 683 (1994).

[23] P.V. Landshoff, Phys. Lett. B 386, 291 (1996).

[24] R.E. Cutkosky, J. Math. Phys. 1, 429 (1960).

[25] R.J. Eden, P.V. Landshoff, D.I. Olive and J.C. Polkinghorne, The analytic S-matrix, Cambridge University Press (1966).

[26] R.L. Kobes and G.W. Semenoff, Nucl. Phys. B 260, 714 (1985).

[27] J.I. Kapusta and S.M.H. Wong, Phys. Rev. D 62, 037301 (2000).

[28] H.A. Weldon, Phys. Rev. D 44, 3955 (1991). 
[29] J.I. Kapusta and S.M.H. Wong, Phys. Rev. C 62, 027901 (2000).

[30] R. Kobes, Phys. Rev. D 42, 562 (1990).

[31] R. Kobes, Phys. Rev. D 43, 1269 (1991).

[32] T.S. Evans, Phys. Lett. B 249, 286 (1990).

[33] P. Aurenche and T. Becherrawy, Nucl. Phys. B 379, 259 (1992).

[34] J.C. Taylor, Phys. Rev. D 47, 725 (1993).

[35] P.F. Bedaque, A. Das and S. Naik, Mod. Phys. Lett. A 12, 2481 (1997).

[36] F. Gelis, Nucl. Phys. B 508, 483 (1997). 